\title{
Network-coded Cooperation for Multi-unicast with Non-Ideal Source-Relay Channels
}

\author{
Yong Liu*, Wen Chen*†, Jianbo Ji*, and Jietao Zhang $\ddagger$ \\ *Department of Electronic Engineering, Shanghai Jiaotong University, Shanghai, P. R. China, 200240 \\ $\nmid$ †KL for Mobile Communications, Southeast University, P. R. China, 211189 \\ †Communication Technologies Department, Huawei Technologies, CO., Ltd., P. R. China 518129 \\ Email: $\{$ yongliu1982,wenchen\}@sjtu.edu.cn
}

\begin{abstract}
Network coding is considered as a promising technique to improve diversity gain and network throughput in multi-source relay systems. This paper presents an opportunistic network coded cooperation scheme in wireless multi-unicast system with non-ideal source-relay channels. In the conventional network-coding-based cooperation schemes, the relay merges the messages received from multiple sources and always forwards them to the destinations without checking their reception status. The destinations recover the sources' messages either from the direct transmissions or from the relay forwarding. Such mode is easy to implement, but may lead to error propagation in the event of decoding failures at relay. In contrast to these works, the proposed scheme is opportunistic, where the relay forwarding is determined by the quality of $S \rightarrow R$ channels, that is, the relay does not assist transmission unless it has correctly decoded the received sources' messages. Systematic performance analysis in the form of outage probability and spectral efficiency is performed in this paper. Comparisons with the conventional network coded multi-unicast and Incremental Relaying protocol are made under a fixed system energy constraint. The outage results show that the proposed scheme performs better than the conventional network-coded schemes when source-relay links has poor quality. Comparing with Incremental Relaying protocol, the opportunistic scheme achieves a reduced system outage probability as well as a higher spectral efficiency. This scenario can be extended to general multi-user environment without much cost.
\end{abstract}

Index Terms-Network coding, spectral efficiency, multiunicast, opportunistic transmission, outage probability.

\section{INTRODUCTION}

With the potential for remarkable benefits in terms of system capacity and diversity gain, cooperative relaying, who has been extensively investigated in [1]-[4], is proposed as an effective means to improve system performance for future generations of wireless networks. In a typical relay system setup, the source broadcasts a message to the destination as well as relay in the first phase; the relay, who is always located between the source and destination, forwards the received signal to the destination in the second phase. The destination recover the sources message based on the two phases' transmission. Depending on the channel state of the network, various forwarding strategies can be employed to exploit the potential benefits provided by node cooperation, among which Amplify-and-Forward (AF) and Decode-andForward (DF) were introduced and carefully examined in [3]. In a relay cooperation system using $\mathrm{AF}$ technology, the relay node, who acts as a virtual antenna, overhears the signal, amplifies and forwards it to the destination, whereas in a DF scheme, the relay node first decodes the received message, re-encodes and forwards it to the destination. Transmission diversity is thus achieved via the two methods, which enables the network to combat channel fading. Similar to the single source-destination pair, multiple source-destination pair can also derive benefit from one relay node if all the terminals lies in the coverage of the relay [5], [6], among which Multiple Access Relay Channel (MARC) is a typical setup.

Network coding, where the packets of many separate messages flows can be mixed via algebraic operations [7], [8], has drawn significant attention for its potential in achieving splendid throughput increases. The core of network coding technology is to allow and encourage data merging in the intermediate nodes to save the system resources such as bandwidth, power, and time slots. Although network coding is first introduced for wired network, many works have proved that it is also beneficial in wireless networks [9]-[11]. Network coding is used to increase the efficiency of information exchange in wireless environments was early proposed in [9]. The authors in [11] showed that network coding leads to a better diversity performance in a distributed antenna system (DAS). Actually, how to leverage network coding in wireless physical layer networks for system capacity improvement is still under intensive study recently.

As mentioned above, multi-source systems can derive significant benefits from network coding in terms of throughput and robustness. Therefore, there have been several proposals for applying network coding to multi-sources relaying systems [12]-[16]. In [12] and [14], an XOR-based network coding scheme is employed to facilitate the bi-directional information exchange between a pair of nodes over two hops. In [13], in order to improve both the reliability and throughput for a two-stage DF network with multiple source-destination pairs, network coding is combined with Best-Select cooperation which is performed by exploiting the instantaneous channel state information (CSI). In [15]and[16], networkcoding-based MARC is proved maintaining the diversity gain, because the broadcast nature of wireless networks is fully exploited to provide a flexible transmission in the schemes.

The aforementioned works were mainly aimed at improving the performance of a multi-source network with stationary traffic flows. However, fading nature is stochastic in wireless 
networks. Sometimes channels condition between the sources and relay may not so good to guarantee reliable decoding. In such case, network-coded cooperation performed by the relay would lead to error propagation. Based on this fact, we propose an Opportunistic Network-Coded (ONC) multi-unicast scheme in this paper, where the relay node decides whether or not to transmit the network-coded information based upon the quality of $S \rightarrow R$ channels. In other words, the relay will do nothing unless it has correctly decoded the transmitted messages. The concept here is similar to the Incremental Relaying mentioned in [3], but network coding technology is not adopted in that scheme.

The advantages of our work over the existing cooperation protocols are shown in two aspects. On one hand, the proposed scheme utilizes opportunistic relaying scheme, where the relay does not cooperate unless it has correctly decoded some messages form the sources. Due to exploitation of opportunistic relaying, the new scheme improves spectral efficiency like incremental relaying by avoiding unnecessary relaying. This property realizes more efficient use of the degrees of freedom of the channel than the existing protocols where the relay always perform cooperation. On the other hand, network coding technology is adopted, which overall reduces time slots compared with those not exploiting network coding. As a consequence, with the same system energy constraint, each transmit node will be allocated with much more transmit power.

In this paper, we perform outage and spectral efficiency analysis of the network-coding-based multi-unicast system. To make a fair comparison, we assume that the same bandwidth and transmit power are allocated in all setups, which however were missing in the previous works. Compared with Incremental Relaying scheme that does not exploit network coding, the new scheme provides a higher spectral efficiency while maintaining a slightly reduced system outage probability. We especially compare the satic and opportunistic networkcoding-based schemes.

The rest of this paper is organized as follows. Section II describes the ONC system model. In Section III, outage probability, and spectral efficiency for the proposed scheme are analyzed. In Section IV, performance evaluation are presented to verify the performance improvement of the new scheme. Section VI concludes the paper.

\section{SYSTEM MOdEL}

\section{A. Channel Model}

The multi-unicast system considered in this paper is depicted in Fig. 1, which consists of five nodes, where two sources $S_{a}$ and $S_{b}$ communicate with two destination nodes $D_{p}$ and $D_{q}$ assisted by one half duplex relay $R$. The channels are assumed to be flat-fading, mutually spatially independent, with additive white Gaussian noise (AWGN) and modeled as

$$
y_{i, j}=h_{i, j} x_{i, j}+n_{i, j},
$$

where $h_{i, j}$ represents the fading gain of the corresponding channel, in which $i \in\{a, b, r\}$ and $j \in\{r, p, q\}, x_{i, j}$ and $y_{i, j}$ are the inputs and outputs of the channel, $n_{i, j}$ is the

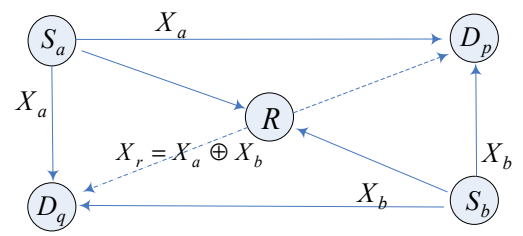

Fig. 1. Multi-unicast model with two sources-destination pairs.

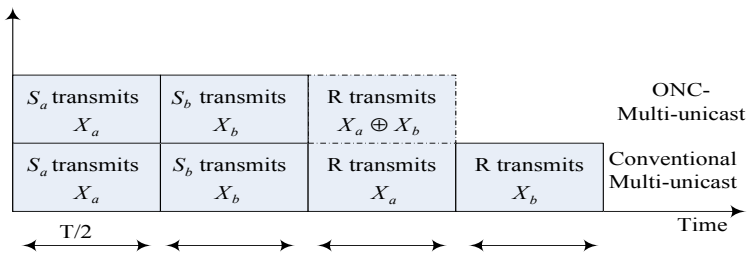

Fig. 2. Timing channel allocation of the conventional and the proposed network-coding-based multi-unicast system.

additive Gaussian white noise with variance $N_{0}$. Channels are modeled as zero-mean, circularly symmetric complex Gaussian random variables with variances $1 / \alpha_{i, j}$. Hence $\left|h_{i, j}\right|$ is Rayleigh distributed and $\left|h_{i, j}\right|^{2}$ is exponentially distributed with exponent $\alpha_{i, j}$ respectively.

In addition, all the transmit terminals are assumed to have a common transmit power denoted as $P$. Then the instantaneous SNR of the channel can be denoted as $\gamma_{i, j}=\left|h_{i, j}\right|^{2} \frac{P}{N_{0}}$. As all nodes work in the half-duplex mode, transmission orthogonality should be satisfied.

In the conventional multi-unicast cooperation schemes, a source transmits a signal $x_{i}$ in the first half time slot. Then the relay forwards the same information in the second half time slot employing AF or DF scheme in TDMA mode. The proposed network coding based transmission scheme, however, realize network coding and spectral efficiency simultaneously. In the new scheme, the source transmits two different symbols $x_{a}$ and $x_{b}$ in the first two half time slots respectively. Then the relay may forward the XOR $x_{a} \bigoplus x_{b}$ to the destinations in third half time slot, as shown in Fig. 2. This scheme may save $1 / 4$ time slot compared with the conventional scheme, and improve the spectral efficiency as well as power efficiency.

\section{B. Description of ONC-Multi-Unicast Scheme}

In this subsection, the operation of ONC-multi-unicast Scheme will be described in details. Due to the exploitation of opportunistic relaying, the proposed scheme improves spectral efficiency as incremental relaying scheme in [3], since the relay is only employed when helpful.

During the first two half time slots, $S_{a}$ and $S_{b}$ transmit their own data towards the distinct destination nodes as well as the relay at spectral efficiency $R$ bit $/ \mathrm{s} / \mathrm{Hz}$ respectively. Based on decoding result, the relay chooses to work either in a static or a opportunistic mode [3]. In the static mode, the relay decodes the messages and performs the network coding without checking the decoding status. Apparently, if at least one of the two messages is incorrectly decoded, the network-coded message does not help in recovering the sources' messages at the destination. Such mode is simple to implement, but may 
lead to error propagation; While in the opportunistic mode, the relay does not perform network coding unless both sources' transmissions are correctly decoded. If the received SNR at $R$ from $S_{i}$ is not high enough to guarantee successful decoding, the relay will not perform network coding. It either forwards the correctly decoded one of the two messages (if any), or keeps idle. For clarity, we broadly categorize the opportunistic protocols into three disjoint cases as follows.

1) The relay correctly decodes both of the two sources' messages. Then the relay performs the network coding and forwards the network-coded message to destinations in the third half time slot.

2) The relay failed to decode any of $X_{a}$ and $X_{b}$. Then the relay keeps idle in the third half time slot.

3) The relay succeeds in decoding only one of the two sources' messages. Then it merely forwards the correctly decoded message in the third half time slot.

\section{PERFORMANCE ANALYSIS AND IMPROVEMENTS}

Outage probability is an analytical measurement for block fading environment, which denotes the probability of event that the instantaneous mutual information $I$ is smaller than the data rate $R$. Consider the point-to-point transmission of $S_{a}$ to the destination $D_{p}$ for example. When the instantaneous SNR of the channel $\gamma_{a, p}=\left|h_{a, p}\right|^{2} \frac{P_{t}}{N_{0}}$ is less than some threshold, the destination $D_{p}$ will wrongly decode $X_{a}$. Then an outage is said to occur, and the outage probability is

$$
P\left(\bar{X}_{a}\right)=P\left(\gamma_{a, p}<2^{R}-1\right)=1-\exp \left(-\frac{2^{R}-1}{\Gamma_{a, p}}\right),
$$

where $\bar{X}_{a}$ represents $X_{a}$ in outage, the channel coefficient $\left|h_{a, p}\right|^{2}$ is exponentially distributed, and $\Gamma_{a, p}$ is the average SNR of the channel $h_{a, p}$.

According to the decoding status at the relay, there are several possibilities of transmission. We will analyze the outage probability of the network-coding-based multi-unicast system for each case in the following. Without loss of generality, we only consider the outage event of the signal $X_{a}$ at destination $D_{p}$, which can be easily modified to other events.

Let $X_{i}^{(j, k)}$ be the correctly decoded message of source $i$ transmitted from node $j$ to node $k$; while $\bar{X}_{i}^{(j, k)}$ denotes the corresponding outage.

\section{A. Relay operating in static mode}

In this mode, relay try to decode the messages and always performs network coding without checking the decoding status. Depending on the quality of $S_{i} \rightarrow R$ channels, the outage computation can be classified into two cases.

CASE 1: The relay succeed in decoding both sources messages, and forwards the network coded symbol $X_{a \oplus b}=$ $X_{a} \oplus X_{b}$ to the destinations. Then the outage event of source $S_{a}$ is

$$
\bar{X}_{(a, 1)}=X_{a}^{(a, r)} \cdot X_{b}^{(b, r)} \cdot \bar{X}_{a}^{(a, p)} \cdot\left(\bar{X}_{b}^{(b, p))}+\bar{X}_{a \oplus b}^{(r, p)}\right),
$$

where ${ }^{\prime}+{ }^{\prime}$ and ${ }^{\prime} \cdot{ }^{\prime}$ represent the logical ' $o r^{\prime}$ and ' $a n d^{\prime}$ operators respectively, and the subscript 1 denotes the case 1 . Because the broadcast nature of wireless communication, $D_{p}$ can overhear $X_{b}$ at the first half time slot, and $D_{q}$ can overhear $X_{a}$ at the second half time slot. So even if $X_{a}$ sometimes fails to be received from the direct transmission, the destination $D_{p}$ is also able to recover the message by combining $X_{b}$ and $X_{a \oplus b}$, provided that both $X_{b}$ and $X_{a \oplus b}$ are correctly received. Therefore, we can write the system outage event $\bar{X}_{s}$ as

$$
\begin{gathered}
\bar{X}_{(s, 1)}=X_{a}^{(a, r)} \cdot X_{b}^{(b, r)} \cdot \bar{X}_{a}^{(a, p)} \cdot\left(\bar{X}_{b}^{(b, p))}+\bar{X}_{a \oplus b}^{(r, p)}\right) \\
+X_{a}^{(a, r)} \cdot X_{b}^{(b, r)} \cdot \bar{X}_{b}^{(b, q)} \cdot\left(\bar{X}_{b}^{(a, q))}+\bar{X}_{a \oplus b}^{(r, q)}\right) .
\end{gathered}
$$

CASE 2 : At least one signal is wrongly decoded at the relay. In this case, the transmission by the relay node does not help in message recovery at the destinations, and the outage events at the destination merely depend on direct transmission. Then the outage of $X_{a}$ is

$$
\bar{X}_{(a, 2)}=\left(\bar{X}_{a}^{(a, r)}+\bar{X}_{b}^{(b, r)}\right) \cdot \bar{X}_{a}^{(a, p)}
$$

Therefore the system outage in case 2 is

$$
\bar{X}_{(s, 2)}=\left(\bar{X}_{a}^{(a, r)}+\bar{X}_{b}^{(b, r)}\right) \cdot\left(\bar{X}_{a}^{(a, p)}+\bar{X}_{b}^{(b, q)}\right) .
$$

Because of the independence of outage events, the system outage probability is

$$
\begin{aligned}
P\left(\bar{X}_{(s)}\right)= & P\left(X_{a}^{(a, r)}\right) \cdot P\left(X_{b}^{(b, r)}\right) \cdot P\left(\bar{X}_{a}^{(a, p)}\right) \\
& \cdot P\left(\bar{X}_{b}^{(b, p))}+\bar{X}_{a \oplus b}^{(r, p)}\right) \\
& +P\left(X_{a}^{(a, r)}\right) \cdot P\left(X_{b}^{(b, r)}\right) \cdot P\left(\bar{X}_{b}^{(b, q)}\right) \\
& \cdot P\left(\bar{X}_{b}^{(a, q))}+\bar{X}_{a \oplus b}^{(r, q)}\right) \\
& +P\left(\bar{X}_{a}^{(a, r)}+\bar{X}_{b}^{(b, r)}\right) \cdot P\left(\bar{X}_{a}^{(a, p)}+\bar{X}_{b}^{(b, q)}\right),
\end{aligned}
$$

where all individual probabilities in (7) can be computed by (2).

\section{B. Relay operating in opportunistic mode}

Similarly, there are several possibilities of forwarding actions depending on the decoding results at the relay node. In the following, we will analyze the outage probability for each case.

CASE 1 : Both sources messages are lost at the relay. Then the relay keeps idle. Hence the outage events at the destination $D_{p}$ is decided by the direct transmissions only, that is

$$
\bar{X}_{(p, 1)}=\bar{X}_{a}^{(a, r)} \cdot \bar{X}_{b}^{(b, r)} \cdot \bar{X}_{a}^{(a, p)},
$$

Then the system outage event is

$$
\bar{X}_{(s, 1)}=\bar{X}_{a}^{(a, r)} \cdot \bar{X}_{b}^{(b, r)} \cdot\left(\bar{X}_{a}^{(a, p)}+\bar{X}_{b}^{(b, q)}\right) .
$$

CASE 2 : The relay only correctly decodes one message from the two sources. Without loss of generality, we only 
consider the relay correctly decodes message from $S_{a}$, but fails to $X_{b}$. In this case, the relay just forward $X_{a}$ instead of performing network coding. Then the outage event becomes

$$
\bar{X}_{(a, 2)}=X_{a}^{(a, r)} \cdot \bar{X}_{b}^{(b, r)} \cdot \bar{X}_{a}^{(C)},
$$

where $X_{a}^{(C)}=\operatorname{MRC}\left(X_{a}^{(a, p)}, X_{a}^{(r, p)}\right)$ represents the combined message at the destination. The outage event of $D_{q}$ can be obtained similarly, and the system outage event in case 2 is

$$
\begin{aligned}
\bar{X}_{(s, 2)}= & X_{a}^{(a, r)} \cdot \bar{X}_{b}^{(b, r)} \cdot\left(\bar{X}_{a}^{(C)}+\bar{X}_{b}^{(b, q)}\right) \\
& +X_{b}^{(b, r)} \cdot \bar{X}_{a}^{(a, r)} \cdot\left(\bar{X}_{b}^{(C)}+\bar{X}_{a}^{(a, p)}\right) .
\end{aligned}
$$

CASE 3 : Both of the messages are correctly decoded at relay. This is the same as the first case of the static mode, and equation (3) and (4) can be applied here. Therefore, the system outage probability in the opportunistic mode can be expressed as

$$
\begin{aligned}
P\left(\bar{X}_{(s)}\right)= & P\left(X_{a}^{(a, r)}\right) \cdot P\left(X_{b}^{(b, r)}\right) \cdot P\left(\bar{X}_{a}^{(a, p)}\right) \\
& \cdot P\left(\bar{X}_{b}^{(b, p))}+\bar{X}_{a \oplus b}^{(r, p)}\right) \\
& +P\left(X_{a}^{(a, r)}\right) \cdot P\left(X_{b}^{(b, r)}\right) \cdot P\left(\bar{X}_{b}^{(b, q)}\right) \\
& \cdot P\left(\bar{X}_{b}^{(a, q))}+\bar{X}_{a \oplus b)}^{(r, q)}\right) \\
& +P\left(\bar{X}_{a}^{(a, r)}\right) \cdot P\left(\bar{X}_{b}^{(b, r)}\right) \cdot P\left(\bar{X}_{a}^{(a, p)}+\bar{X}_{b}^{(b, q)}\right) \\
& +P\left(X_{a}^{(a, r)}\right) \cdot P\left(\bar{X}_{b}^{(b, r)}\right) \cdot P\left(\bar{X}_{a}^{(C)}+\bar{X}_{b}^{(b, q)}\right) \\
& +P\left(X_{b}^{(b, r)}\right) \cdot P\left(\bar{X}_{a}^{(a, r)}\right) \cdot P\left(\bar{X}_{b}^{(C)}+\bar{X}_{a}^{(a, p)}\right),
\end{aligned}
$$

where $P\left(\bar{X}_{a}^{(C)}\right)$ and $P\left(\bar{X}_{b}^{(C)}\right)$ are the CDFs of the sum of two exponentially distributed random variables, which is given by equation (14). In equation (14), $\lambda_{u}=\frac{1}{\Gamma_{a, p}}, \lambda_{v}=\frac{1}{\Gamma_{r, p}}$, and $\omega=2^{2 R}-1$ is the threshold [3]. Then we can use equation (2) and (14) to evaluate the individual probabilities accordingly.

\section{Numerical Results}

In this section, we present some numerical results to evaluate the proposed scheme. We use monte-carlo simulation in Matlab for $10^{6}$ runs to estimate the outage probability for the new scheme. Besides, the conventional multi-unicast and the Incremental Relaying schemes in [3] are also presented for comparison. An information rate of $R=1$ is assumed. Moreover, results are based on symmetric source-destination and source-relay channels, that is, $\Gamma_{a, p}=\Gamma_{b, q}, \Gamma_{a, r}=\Gamma_{b, r}$. Apparently, the selection of operation modes mainly depends on the quality of the source-relay links $\Gamma_{i, r}$.

In order to illuminate the superiority of the opportunistic network coding, we first exhibit the outage probability curves of static protocol with and without network coding, which is

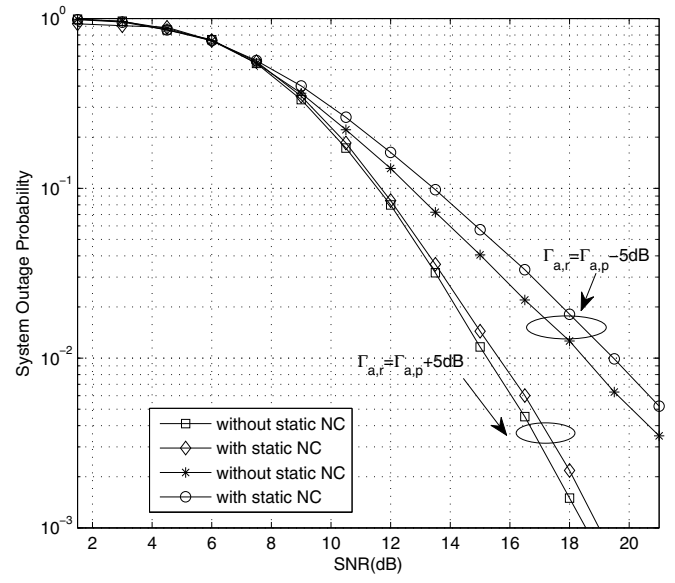

Fig. 3. Outage probability vs SNR of static protocol with and without network coding. NC is short for Network coding, and results are based on 2 different values of $\Gamma_{i, r}$

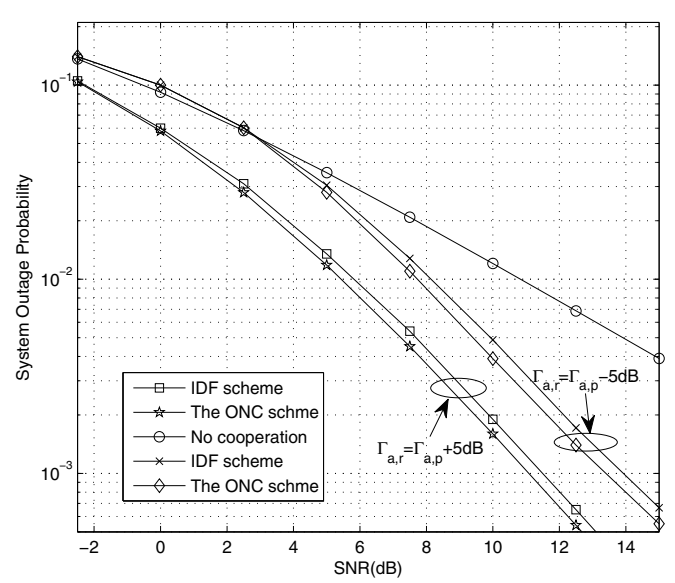

Fig. 4. Outage probabilities of IDF and opportunistic network-coding-based protocols. IDF stands for Incremental Decode-and-Forward, ONC is short for Opportunistic Network-Coding

shown in Fig. 3. We can find an interesting phenomenon that the protocol without network coding always performs better compared to that in static mode. So it is not necessary to employ network coding in static modes in terms of outage probability even though the protocols are relatively simple to implement. Fig. 4 shows the outage probabilities of IDF and the opportunistic network-coding-based protocols. It is easy to find that the opportunistic network-coding scheme maintains a lower system outage probability than IDF. Actually a stable SNR gain of $0.5 \mathrm{~dB}$ is achieved by the new scheme compared with IDF.

Next, we study the spectral efficiencies of IDF and the opportunistic network-coding-based protocols. Apparently, both of the two schemes are rateless, because they do not always perform message forwarding. Based on the analysis in Section III, expected spectral efficiency of ONC-based protocol 


$$
P\left(\bar{X}_{a}^{(C)}\right)=P\left(\gamma_{a, p}+\gamma_{r, p}\right)<2^{R}-1=\left\{\begin{array}{cc}
1-\left[\left(\frac{\lambda_{u}}{\lambda_{u}-\lambda_{v}}\right) \cdot e^{\left(-\lambda_{v} \omega\right)}+\left(\frac{\lambda_{v}}{\lambda_{v}-\lambda_{u}}\right) \cdot e^{\left(-\lambda_{u} \omega\right)}\right], & \lambda_{u} \neq \lambda_{v} \\
1-\left(1+\lambda_{u} \omega\right) \cdot e^{\left(-\lambda_{u} \omega\right)} & , \quad \lambda_{u} \neq \lambda_{v}
\end{array}\right.
$$

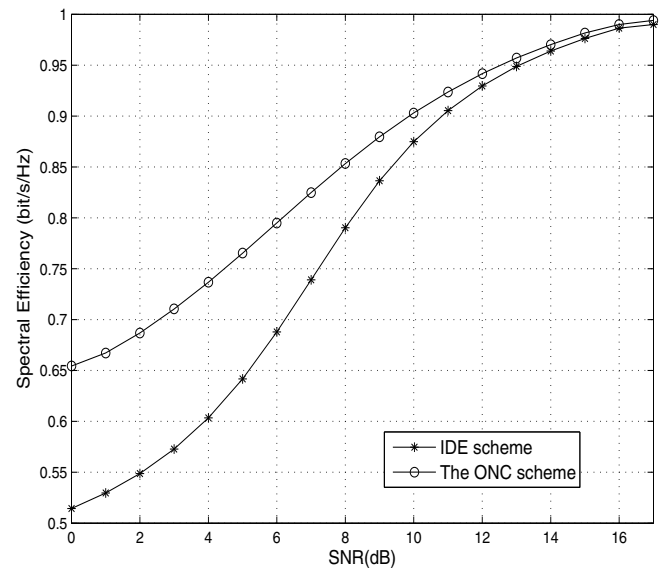

Fig. 5. Spectral Efficiency comparison of IDF and opportunistic networkcoding-based protocols. $R=1 \mathrm{~b} / \mathrm{s} / \mathrm{Hz}$.

is

$$
\begin{aligned}
S^{O N C}= & R \cdot P\left\{\gamma_{a, p}<2^{R}-1\right\} \\
& +\frac{2 R}{3} P\left\{\gamma_{a, r}<2^{R}-1\right\} P\left\{\gamma_{b, r}<2^{R}-1\right\} \\
& +\frac{R}{2} P\left\{\gamma_{a, r}>2^{R}-1\right\} P\left\{\gamma_{b, r}<2^{R}-1\right\}
\end{aligned}
$$

while that of IDF protocol elaborated in [3] is,

$$
\begin{aligned}
S_{i}^{I D F}= & R \cdot \exp \left(-\alpha_{i j}\left(2^{R}-1\right) P / 4 N_{0}\right) \\
& +\frac{R}{2}\left(1-\exp \left(-\alpha_{i j}\left(2^{R}-1\right) P / 4 N_{0}\right)\right),
\end{aligned}
$$

where $i \in\{a, b\}$ and $j \in\{p, q\}$.

We can see that the opportunistic network-coding-based protocol exceeds the IDF protocol in terms of spectral efficiency. Moreover, both IDF and the proposed scheme provide an expected spectral efficiency approaching $R(=1 \mathrm{bit} / \mathrm{s} / \mathrm{Hz})$ as $S N R_{\left(s_{i}, d_{j}\right)}$ approaches to $\infty$, which can be understood intuitively.

Based on these results, one can observe that compared with the schemes without network coding and IDF, the opportunistic network-coding-based protocol provides a reduced system outage probability while maintaining an increased expected spectral efficiency, which comes from the employment of the network coding technology and the opportunistic forwarding.

\section{CONCLUSION}

In this paper, we propose an opportunistic network-codingbased multi-unicast protocol, and present the outage probability and spectral efficiency analysis of various cooperative transmission schemes including the conventional multi-unicast and the IDF scheme. By employing network coding technology, the time slots are reduced in the transmission, which can be translated into the higher spectral efficiency and increased transmit power. On the other hand, by employing opportunistic scheme in the protocol, unnecessary forwarding by relay is avoided, which results in efficient use of the degrees of freedom of the channel. We proved that our scheme outperforms IDF in terms of system outage behavior and spectral efficiency via theoretical analysis and numerical results. Moreover, we observed that the static protocols do not benefit from network coding in terms of outage probability.

\section{ACKNOWLEDGEMENT}

This work is supported by NSF China \#60972031, by SEU SKL project \#W200907, by Huawei Funding \#YJCB2009024WL and \#YJCB2008048WL, and by National 973 project \#2009CB824900.

\section{REFERENCES}

[1] G. Kramer, M. Gastpar, and P. Gupta, "Cooperative Strategies and Capacity Theorems for Relay Networks," IEEE Trans. Inf. Theory, vol. 51, no. 9, pp. 3037-3062, Sept., 2005.

[2] P. Herhold, "Cooperative relaying protocols and performances," $\mathrm{PhD}$ thesis at the Technical University of Dresden, July, 2005.

[3] J. L. Laneman, D. N. C. Tse, and G. W. Wornell, "Cooperative diversity in wireless networks: efficient protocols and outage behavior," IEEE Trans. Inf. Theory, Vol. 50, No. 12, pp. 3062-3080, Dec., 2004.

[4] Y. Liang, and G. Kramer, "Rate regions for relay broadcast channels," IEEE Trans. Inf. Theory, vol. 53, no. 10, pp. 3517-3535, Oct., 2007.

[5] L. Sankar, Y. Liang, H. V. Poor, N. Mandayam, "Opportunistic Communications in an othogonal Multiaccess Relay Channel," IEEE International Symposium on Information Theory, Nice, France, June 24-29, 2007.

[6] O. Sahin, E. Erkip, "Dynamic resource allocation for multi sourcedestination relay networks," CISS '07, pp. 19-24, March, 2007.

[7] R. Ahlswede, N. Cai, R. Li, and R. Yeung, "Network information flow", IEEE Trans. Inf. Theory, vol. 46, no. 4, pp. 1204-1216, July, 2000.

[8] R. Li, R. Yeung, and N. Cai, "Linear network coding," IEEE Trans. Inf. Theory, vol. 49, no. 2, pp. 371-381, Feb., 2003.

[9] Y. Wu, P. A. Chou, and S.-Y. Kung, Information exchange in wireless networks with network coding and physical-layer broadcast, Microsoft Research Technical Report MSR-TR-2004-78, Aug., 2004.

[10] J. Zhang, P. Fan, and K. B. Letaief, "Network Coding for Efficient Multicast Routing in Wireless Ad-hoc Networks," IEEE Trans. Com., no.4, vol. 56, pp. 598-607, Apr., 2008.

[11] Yingda, S. Kishore, and J. T. Li, "Wireless Diversity through Network Coding," Proc.IEEE WCNC., vol. 3, pp. 1681-1686, April, 2006.

[12] Y. Wu, P. Chou, and S. Kung, "Information exchange in wireless networks with network coding and physical-layer broadcast," in Proc. of CISS 2005, March 16-18, 2005.

[13] C. Peng, Q. Zhang, M. Zhao, and Y. Yao, "SNCC: A selective networkcoded cooperation scheme in wireless networks," in Proc. of ICC 2007, pp. 4219-4224, June, 2007.

[14] W. Chen, K. Letaief, and Z. Chao, "Opportunistic network coding for wireless networks," in Proc. of ICC 2007, pp. 4634-4639, June, 24-28, 2007.

[15] C. Hausl, and P. Dupraz, "Joint network-channel coding for the multiple access relay channel," in Proc. Intern. Workshop on Wireless Ad Hoc and Sensor Networks, New York, USA, vol. 3, pp. 817-822, June, 2006.

[16] D. H. Woldegebreal, and H. Karl, "multiple-access relay channel with network coding and Non-ideal source-relay channels," in Proc. IEEE ISWCS, pp. 732-736, Oct., 2007. 